\title{
A-08
}

\section{INFLUENCIA DEL RIEGO DEFICITARIO CONTROLADO PRECOSECHA SOBRE LA CALIDAD DE LA CEREZA 'PRIME GIANT'}

\author{
Blanco Montoya, $V^{1}{ }^{1}$, Domingo Miguel, $R^{1}{ }^{1}$, Artés Hernández $F^{2}{ }^{2}$, García Riquelme, M. ${ }^{1}$, \\ Pérez Pastor, A. ${ }^{1}$, Blaya Ros P.J. ${ }^{1}$, Torres Sánchez, R. $^{3}$
}

${ }^{1}$ Dpto. de Producción Vegetal. Universidad Politécnica de Cartagena (ETSIA-UPCT). Paseo Alfonso XIII, 48. 30203 Cartagena. victor.blanco@upct.es; rafael.domingo@upct.es; manuel.garcia@upct.es; alex.perez-pastor@upct.es; p.blaya18@gmail.com.

${ }^{2}$ Dpto. Ingeniería de los Alimentos y del Equipamiento Agrícola. Universidad Politécnica de Cartagena (ETSIA-UPCT). fr.artes-hdez@upct.es.

${ }^{3}$ Dpto de Ingeniería de Sistemas y Automática. Universidad Politécnica de Cartagena (ETSIIUPCT).roque.torres@upct.es.

\section{Resumen}

El uso de redes de sensores para el manejo del riego deficitario controlado (RDC) en cerezo puede incidir en la mejora de la productividad del agua, calidad de la fruta y en su comportamiento poscosecha, al permitir el conocimiento y control del estado hídrico del suelo y árbol en todo momento.

En el primer año de ensayo, el riego deficitario aplicado en precosecha para satisfacer el $85 \%$ de las necesidades máximas del cultivo $\left(\mathrm{ET}_{\mathrm{cg}}\right)$ permitió un ahorro de agua del $17 \%\left(380 \mathrm{~m}^{3} \mathrm{ha}^{-1}\right)$ respecto al tratamiento control, que se regó al $110 \%$ de la $\mathrm{ET}_{\text {cg. Este }}$ déficit hídrico ligero no afectó a la producción $\left(16,13 \mathrm{t} \mathrm{ha}^{-1}\right)$ e incluso mejoró la calidad del fruto. Así, en el momento de la cosecha $\left(\mathrm{t}_{0}\right)$ los frutos bajo déficit ligero presentaron matices más rojos y mayor acidez que los de riego completo. Esta mayor acidez no afectó al índice de madurez debido a la compensación por sólidos solubles totales (SST). El carácter de mayor acidez perduró tras 30 días de conservación en frio $\left(\mathrm{t}_{1}\right)$ y 5 días de simulación de las condiciones de comercialización $\left(\mathrm{t}_{2}\right)$. Igualmente, los frutos bajo déficit hídrico presentaron una tendencia a menores pérdidas de peso por deshidratación a finales de los periodos de conservación en frio y de simulación de la comercialización.

\section{1- Introducción}

La situación permanente de escasez de agua en la agricultura de regadío de las zonas semiáridas ha propiciado el uso y puesta a punto de nuevas tecnologías aplicadas al riego, mejorando la eficiencia en la distribución, transporte y aplicación del agua a nivel de parcela. Sin embargo, el ahorro de agua derivado de ellas sigue siendo insuficiente para satisfacer las necesidades de agua de sus sectores productivos. De ahí, la necesidad de desarrollar nuevas alternativas que redunden en la mejora de la gestión del agua. Una alternativa que puede contribuir a paliar esta situación de escasez en sector agrícola es el empleo de estrategias de riego deficitario controlado (Ruiz-Sanchez et al., 2010). Así, distintos estudios realizados en frutales y vid demuestran que algunas especies son capaces de tolerar déficits hídricos en determinados períodos del ciclo de cultivo sin mermas de producción ni de calidad, como es el caso del limonero (Domingo et al., 1994), almendro (Egea et al., 2012; Puerto et al., 2015), nectarina (De la Rosa et al., 2016), etc.

El empleo de este tipo de estrategias no sólo ha dado lugar a un aumento de la productividad del agua sino que en muchos casos han mejorado la calidad y comportamiento poscosecha de los frutos; entre ellos del albaricoque (Pérez-Pastor et al., 2007), uva de mesa (Conesa et al., 2015) y melocotón (Falagán et al., 2015). 
Este trabajo se realizó en cerezo un cultivo prometedor por sus elevados precios de mercado, por ser una alternativa interesante a otros cultivos, ya que permite simultanear su manejo con el de otros frutales de la explotación. La cereza es un fruto no climatérico, muy perecedero, delicado y al mismo tiempo muy apreciado por el consumidor. Temperaturas próximas a $0^{\circ} \mathrm{C}$ y alta humedad relativa (90-95\%) permiten mantener su calidad, retrasando al máximo las pérdidas por deshidratación. Sin embargo, la refrigeración por períodos extensos puede provocar pérdida de color, acidez, aromas, y favorecer el desarrollo de enfermedades fúngicas.

El objetivo de este estudio fue evaluar la influencia de un déficit hídrico ligero en precosecha sobre la producción y las características físico-químicas de la cereza 'Prime Giant', una variedad de maduración media, en el momento de la cosecha, tras su conservación refrigerada y después de un periodo de simulación de las condiciones de comercialización.

\section{2- Materiales y métodos}

El ensayo se realizó durante 2015 en una parcela de 0,5 ha perteneciente a la finca comercial "Finca Toli" en Jumilla, Murcia ( $38^{\circ} 8^{\prime} \mathrm{N} ; 1^{\circ} 22^{\prime} \mathrm{W}$ y $680 \mathrm{~m}$ de altitud). El suelo es de textura franco-arenosa y medianamente pedregoso, lo que le confiere unas propiedades de retención de agua medias. La plantación de 15 años de edad, constituida por cerezos [Prunus avium (L.)] cv. "Prime Giant", sobre patrón SL 64 y como polinizadores "Brooks" y "Early Lory", dispuesta a marco de plantación de $5 \mathrm{~m} \times 3 \mathrm{~m}$, está formada en vaso bajo con un área sombreada de aproximadamente el 55\% y diámetro de tronco de $16,3 \mathrm{~cm}$. El diseño del sistema de riego por goteo fue de un único lateral por hilera de árboles con 3 goteros de $4 \mathrm{~L} \mathrm{~h}^{-1}$ por árbol. El agua de riego, de pozo, es de buena calidad y presentó una conductividad eléctrica de $0,8 \mathrm{dS} \mathrm{m}^{-1}$ y un contenido en sodio y cloruros de 79,1 y $120,5 \mathrm{mg}$ $\mathrm{L}^{-1}$, respectivamente. La evapotranspiración de referencia $\left(E T_{0}\right)$ acumulada en el periodo 85161 DDA (día del año) fue $361,6 \mathrm{~mm}$, la $\mathrm{ET}_{0}$ media diaria en precosecha fue de 4,7 mm, y la precipitación total $38,9 \mathrm{~mm}$. La temperatura media diaria tuvo un valor promedio de $16,5^{\circ} \mathrm{C}$, con un valor máximo de $28^{\circ} \mathrm{C}$ (DDA 134), ese mismo día se dio el valor máximo de déficit de presión de vapor (DPV) en precosecha, $2,31 \mathrm{kPa}$, aunque la media diaria para el periodo de estudio fue $0,92 \mathrm{kPa}$. Tanto la fertilización como el control fitosanitario se realizaron de acuerdo con las prácticas seguidas en la explotación. Los frutos se recolectaron en dos fechas, el día 3 de junio (DDA 154) y el 10 de junio (DDA 161) en base al color de la piel y siguiendo las prácticas comerciales habituales.

El ensayo constó de 3 tratamientos de riego : i) control, CTRL, regado para satisfacer el $110 \% \mathrm{ET}_{\mathrm{cg}}$ durante toda la campaña de riegos, ii) agricultor, AGR, programado de acuerdo con su experiencia y iii) riego deficitario controlado, RDC, programado al $85 \%$ de la $E T_{c g}$ durante precosecha, a excepción de los 7 días entre ambas recolecciones que se regó como CTRL. ET $\mathrm{cg}$ se determinó de acuerdo con la expresión propuesta por FAO:

$$
\mathrm{ET}_{\mathrm{cg}}=\mathrm{ET}_{\mathrm{o}} \times \mathrm{K}_{\mathrm{c}} \times \mathrm{K}_{\mathrm{r}}
$$

donde, $\mathrm{ET}_{0}$ es la evapotranspiración de referencia, $\mathrm{K}_{\mathrm{c}}$ el coeficiente de cultivo (Marsal et al. 2010) y $K_{r}$ un coeficiente corrector por localización dependiente de la superficie del suelo cubierta por las copas de los árboles (Fereres y Castel 1981) .

El diseño experimental fue de bloques al azar con 4 repeticiones de 7 árboles cada una. El volumen de agua aplicado se midió con contadores de pulsos (ARAD SF). El potencial hídrico de tallo a mediodía $\left(\Psi_{\mathrm{t}}\right)$ se controló semanalmente a partir de 2 hojas por repetición cercanas a la base del tronco y envueltas con plástico y papel de aluminio durante dos horas (Shackel et al. 1997), utilizando una cámara de presión Soil Moisture Equipment Corp., Model 3005, Santa Barbara,CA, USA).

En la madurez comercial de la fruta, se recogieron y pesaron los frutos provenientes de los 5 árboles centrales de cada repetición. Para ello, se realizaron dos recolecciones en los días del año, DDA, 154 y 161. Así mismo, se contaron los frutos existentes en muestras 
de $5 \mathrm{~kg}$ al objeto de conocer el peso unitario de la cereza. La calidad fisicoquímica de las cerezas de la primera recolección (03/06/2015) se determinó tras la cosecha (tiempo $\left.t_{0}\right)$, tras un periodo de frigoconservación de 30 días a $0^{\circ} \mathrm{C}$ y $90 \%$ humedad relativa $(\mathrm{HR})\left(\mathrm{t}_{1}\right)$, y tras un periodo adicional de simulación de la comercialización de 5 días a $15^{\circ} \mathrm{C}$ y $65 \% \mathrm{HR}\left(\mathrm{t}_{2}\right)$. Para ello, se tomaron muestras de 20 cerezas por repetición y en cuatro repeticiones por tratamiento y día de análisis $\left(\mathrm{t}_{0}, \mathrm{t}_{1}, \mathrm{y} \mathrm{t}_{2}\right)$. Los caracteres geométricos se midieron con un calibre digital, el color se determinó con un colorímetro Minolta CR 300 (Ramsey, NJ, EEUU), la firmeza con un analizador de textura LFRA 1500 (Middleboro, Brookfield, MA, EEUU) con una sonda de $2 \mathrm{~mm}$ de diámetro para rotura y una de 25,42 $\mathrm{mm}$ para compresión, los sólidos solubles totales con un refractómetro (Atago N1, Tokio, Japón) y la acidez titulable por valoración ácido-base.

El análisis de datos se realizó mediante ANOVA y regresiones lineales.

\section{3- Resultados y discusión}

Los volúmenes de riego aplicados en precosecha fueron 2.157, 1.777 y $2.751 \mathrm{~m}^{3} \mathrm{ha}^{-1}$ para CTRL, RDC y AGR respectivamente. El tratamiento AGR recibió un $27,5 \%$ de agua más que CTRL, mientras que la cantidad suministrada a RDC fue un 17,5\% y 35,0\% inferior a la de CTRL y AGR, respectivamente. El potencial de tallo a mediodía $\left(\Psi_{\mathrm{t}}\right)$ presentó valores medios $-0,51,-0,48$ y $-0,57 \mathrm{MPa}$ para CTRL, RDC y AGR, respectivamente. Se dieron diferencias significativas en 4 de los 6 días de medida (Figura 1), principalmente entre los tratamientos más regados (AGR y CTRL) y el deficitario RDC, lo que muestra la sensibilidad de $\Psi_{\mathrm{t}}$ para detectar estreses hídricos ligeros. Si se tiene en cuenta que durante todo el periodo precosecha $\Psi_{\mathrm{t}}$ fue superior o igual a -0,70 MPa (Figura 1), valor por encima del cual consideran que no existe estrés hídrico en la variedad de cereza "Summit" (Marsal et al., 2010), podemos considerar que el estrés aplicado en RDC fue de intensidad ligera.

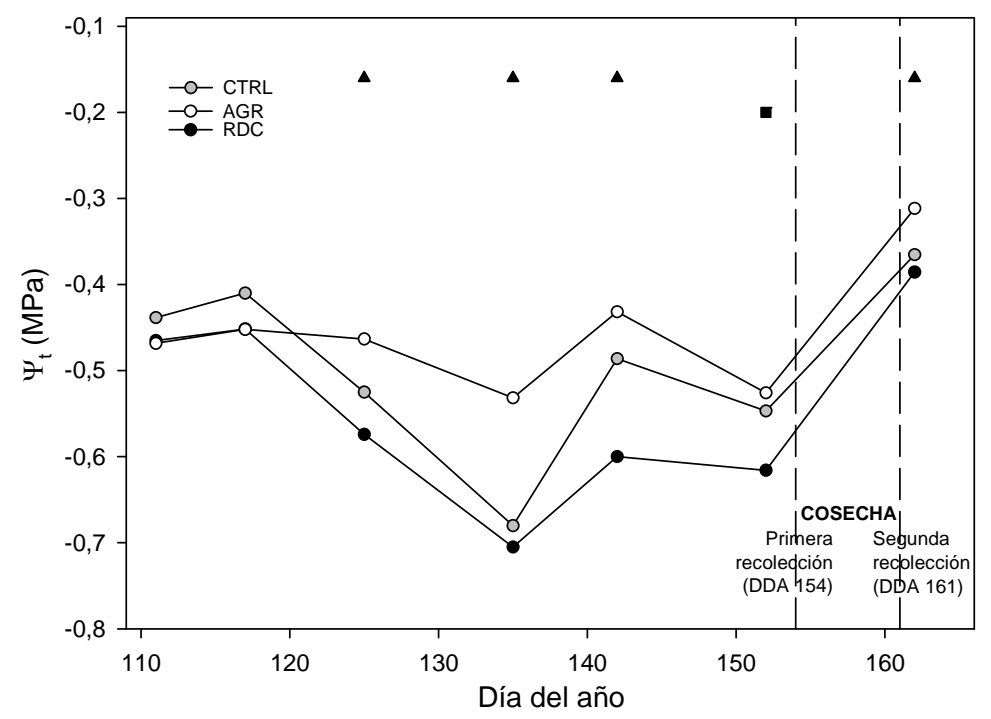

Figura 1. Evolución del potencial de tallo a mediodía en precosecha. Cada punto representa el valor medio de seis medidas. Las diferencias significativas entre tratamientos de acuerdo con el test de Duncan $(P>0,05)$ aparecen indicadas como: diferencias entre 2 tratamientos y $\boldsymbol{\Delta}$ diferencias de un tratamiento con los otros dos.

La tabla 1 muestra la producción total obtenida y la de cada una de las recolecciones. La producción comercial media para el conjunto de tratamientos fue de $15,5 \mathrm{t} \mathrm{ha}^{-1}$ y no hubo diferencias significativas de producción entre tratamientos en ninguna de las dos recolecciones ni en la total. Aunque el peso unitario del fruto fue similar en los tres tratamientos de riego, la tendencia fue a presentar valores más bajos en RDC, viéndose compensada la producción por el mayor número de frutos recolectados. Las producciones 
obtenidas pueden ser consideradas como medias-altas, máxime teniendo en cuenta los daños ocasionados por el granizo, 19 de mayo de 2015, los que fueron cifrados a nivel de parcela entre 1,5 y $2,0 \mathrm{t} \mathrm{ha}^{-1}$ de pérdidas.

Tabla 1. Peso unitario del fruto y producción de cereza por recolección y total.

\begin{tabular}{|c|c|c|c|c|c|}
\hline \multicolumn{2}{|c|}{ Parámetro } & \multicolumn{4}{|c|}{ Tratamiento } \\
\hline & & CTRL & RDC & AGR & \\
\hline Peso unitario fruto & (g) & 11,07 & 10,63 & 11,77 & n.s. \\
\hline Recolección I & $\left(t \cdot h a^{-1}\right)$ & 8,86 & 10,43 & 8,17 & n.s. \\
\hline Recolección II & $\left(t \cdot h a^{-1}\right)$ & 6,72 & 5,71 & 6,67 & n.s. \\
\hline Cosecha Total I+II & $\left(\mathrm{t} \cdot h \mathrm{a}^{-1}\right)$ & 15,58 & 16,13 & 14,84 & n.s. \\
\hline
\end{tabular}

n.s. indica que no existen diferencias significativas entre tratamientos para ese parámetro de acuerdo al test de Duncan $(P>0,05)$.

La tabla 2 recoge los principales parámetros de calidad evaluados en las cerezas de la primera recolección en el momento de la cosecha $\left(\mathrm{t}_{0}\right)$, tras 30 días en cámara frigorífica $\left(\mathrm{t}_{1}\right)$ y tras el periodo de simulación de condiciones de comercialización $\left(t_{2}\right)$. En el momento $t_{0}$, se observaron diferencias significativas entre los tratamientos AGR y RDC en el matiz ('oue) y la acidez titulable (AT). La mayor concentración de ácido málico en RDC no influyó sobre el índice de madurez (IM), al ser ésta compensada por el ligero incremento de sólidos solubles totales (Tabla 2). Las diferencias encontradas en el matiz, pueden deberse a una mayor concentración de antocianinas en el tratamiento RDC, las que provocan un color más intenso. En firmeza no se observaron diferencias entre tratamientos tanto a nivel de compresión como de rotura, aunque sí se dio una relación directa entre la firmeza, especialmente la determinada a compresión, y el agua aplicada a cada tratamiento en precosecha (Figura 2). En cuanto al tamaño del fruto: volumen y diámetro ecuatorial, principales parámetros de calidad de la cereza, no hubo diferencias significativas entre tratamientos y tampoco en el peso unitario del fruto. Sin embargo, se observó una tendencia a mayores pesos unitarios, volumen y diámetro ecuatorial del fruto en los tratamientos más regados en precosecha. La alta correlación encontrada entre el volumen de fruto y el agua aplicada (Figura 2), podría explicar las diferencias encontradas en acidez titulable, y al mismo tiempo, la tendencia de RDC a presentar frutos más pequeños y mayores concentraciones de ácido málico y de antocianinas, relación descrito en uva por Castellarín et al. (2007).

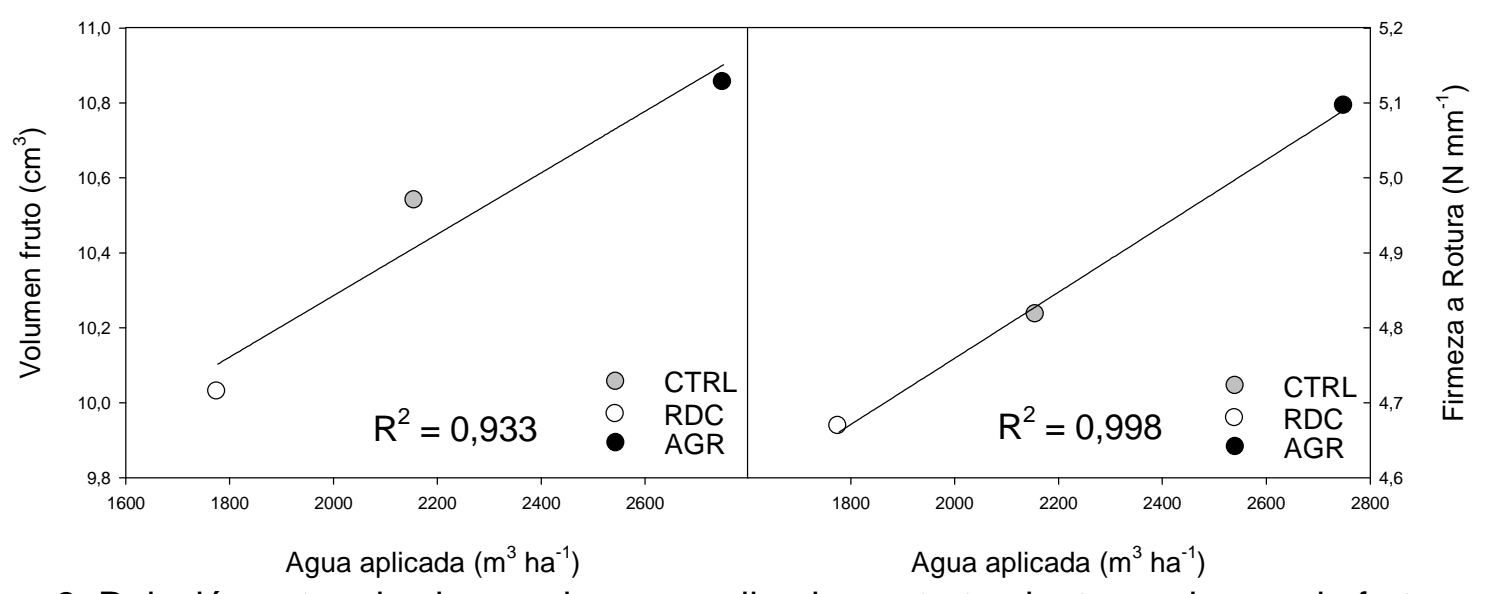

Figura 2. Relación entre el volumen de agua aplicado por tratamiento y volumen de fruto y firmeza a rotura $\left(\mathrm{N} \mathrm{mm}^{-1}\right)$. 
En $t_{1}$, tras 30 días de almacenamiento en cámara frigorífica los parámetros físicos se mantienen constates, y aunque se presupone una pérdida de humedad en la fruta, ésta no ha sido suficiente para inducir un menor volumen o diámetro ecuatorial. Sin embargo, ha promovido un aumento de la concentración de sólidos solubles totales y por consiguiente del valor del índice de madurez. Las diferencias en matiz y acidez titulable que había entre AGR y RDC en el momento de la cosecha desaparecen, experimentando una bajada generalizada de sus valores. De igual modo, la firmeza tanto a compresión como a rotura desciende, particularmente a compresión que sufre una bajada del $22 \%$ de media frente al $14 \%$ a rotura. Ésta pérdida de firmeza tras el almacenamiento en cámara coincide con los resultados publicados por Diaz-Mula et al. (2009). Con respecto al color, la bajada de la saturación y del matiz se debe a un oscurecimiento de las cerezas debido al almacenamiento en cámara frigorífica, informada para la variedad Lapins por Drake y Elfving (2002). Aunque las pérdidas de peso entre tratamientos no fueron significativamente diferentes, se observó una propensión a valores inferiores en RDC (Tabla 2). En albaricoque "Búlida", Pérez-Pastor et al. (2007) informaron que eran los frutos RDC los que sufrían menores pérdidas de peso por deshidratación durante el periodo de frigoconservación.

En $t_{2}$, tras el periodo de 5 días de simulación de condiciones de comercialización los parámetros físicos presentaron valores parecidos a los $t_{1}$ y por tanto a los valores de cosecha. Sin embargo, la firmeza siguió descendiendo, de modo que a compresión se redujo en un $28 \%$ y $8 \%$ respecto a cosecha y frigoconservación, respectivamente. Similares reducciones tuvieron lugar a rotura, cifrándose en un $24 \%$ y $11 \%$ respecto a cosecha y frigoconservación, respectivamente. Aunque la caída de firmeza a compresión en $t_{1}$ parecía más pronunciada que la de rotura, en $\mathrm{t}_{2}$ se iguala, esto puede deberse a que en un principio la pérdida de humedad que ocurre tras el almacenamiento en frío puede endurecer el fruto y dar una sensación de menor pérdida de firmeza. En referencia al color no se encontraron diferencias en el matiz, el cual continuó descendiendo a matices más oscuros en todos los tratamientos. Contrariamente, sí se encontraron diferencias para la saturación entre AGR y los otros dos tratamientos CTRL y RDC. Este parámetro experimentó un considerablemente descenso durante los 5 días de vida comercial, un 30\% de media respecto a $t_{1}$. Los valores de saturación $t_{2}$ corresponden a un rojo oscuro, consecuencia de la rápida pérdida de brillo. La saturación del color de las cerezas puede deberse a un aumento de los procesos de degradación propiciado por las condiciones de conservación, temperatura ambiente de $15^{\circ} \mathrm{C}$ y humedad relativa del 65\%. Esta degradación en el color ha sido igualmente observada en variedades de cereza como Van (Gonçalves et al. 2007). La evolución de los sólidos solubles y la acidez titulable presentó sentidos contrarios, propios de procesos degradativos (Serrano et al. 2009). Así, mientras que los primeros aumentaron, la acidez disminuyó de forma diferencial dando lugar a diferencias significativas entre tratamientos. RDC mantuvo durante el periodo de comercialización mayor nivel de acidez que CTRL y AGR. El índice de madurez (IM) aumentó considerablemente desde cosecha a comercialización en todos los tratamientos, debido principalmente al descenso de la acidez, comportamiento ya descrito en variedades como Brooks o Bing (Crisosto et al. 2002). Aunque la acidez fue superior en RDC, no dio lugar a diferencias en IM entre tratamientos, posiblemente por el ligero repunte experimentado en los sólidos solubles totales (Tabla 2). Aunque los tres tratamientos presentaron similares pérdidas de peso en $\mathrm{t}_{2}$, fue RDC el tratamiento con menor porcentaje de pérdidas, como ya ocurriera en $t_{1}$. Esta propiedad, la cual es muy deseable, puede deberse a pequeños cambios en el fruto, tales como un mayor espesor de cutícula inducido por el déficit hídrico, que disminuirían las pérdidas de agua desde el fruto, Pérez-Pastor et al. (2007). 
Tabla 2. Resultados de los análisis de calidad físico-química de los frutos de la primera recolección.

\begin{tabular}{|c|c|c|c|c|c|c|c|c|c|c|}
\hline \multirow[b]{3}{*}{ Parámetro } & \multirow[b]{3}{*}{ unidad } & \multicolumn{9}{|c|}{ Momento de análisis } \\
\hline & & \multicolumn{3}{|c|}{ Cosecha $\left(t_{0}\right)$} & \multicolumn{3}{|c|}{ Frigoconservación $\left(t_{1}\right)$} & \multicolumn{3}{|c|}{ Comercialización $\left(t_{2}\right)$} \\
\hline & & CTRL & RDC & AGR & CTRL & RDC & AGR & CTRL & RDC & AGR \\
\hline Saturación & croma & 30,7 & 29,04 & 29,73 & 29,27 & 29,61 & 29,85 & $19,18^{b}$ & $20,09^{b}$ & $22,68^{a}$ \\
\hline Matiz & áng hue & $25,89^{a b}$ & $26,81^{a}$ & $25,49^{b}$ & 22,72 & 22,68 & 21,39 & 18,13 & 17,01 & 17,17 \\
\hline Diám. Ecuat. & $\mathrm{mm}$ & 29,59 & 29,13 & 29,95 & 29,52 & 29,22 & 30,00 & 29,43 & 29,25 & 29,25 \\
\hline Volumen & $(\mathrm{cm} 3)$ & 10,54 & 10,03 & 10,85 & 10,54 & 10,36 & 10,97 & 10,56 & 10,46 & 10,31 \\
\hline Pérdida peso & (\%) & & & & 0,70 & 0,44 & 0,81 & 2,23 & 1,50 & 2,41 \\
\hline Firm. Compr. & $\left(\mathrm{N} \mathrm{mm}^{-1}\right)$ & 12,21 & 12,23 & 12,23 & 9,25 & 9,22 & 10,13 & 8,76 & 8,84 & 8,96 \\
\hline Firm. Rotura & $\left(\mathrm{N} \mathrm{mm}^{-1}\right)$ & 4,82 & 4,63 & 5,10 & 4,02 & 4,13 & 4,41 & 3,74 & 3,59 & 3,79 \\
\hline SST & ('Brix) & 18,51 & 18,7 & 18,46 & 18,72 & 19,12 & 19,23 & 19,15 & 20,54 & 19,89 \\
\hline AT & $\left(\mathrm{g} \mathrm{L}^{-1}\right)$ & $9,34^{\mathrm{ab}}$ & $9,83^{a}$ & $9,13^{b}$ & 7,97 & 7,97 & 8,35 & $6,81^{b}$ & $7,28^{a}$ & $6,52^{\mathrm{b}}$ \\
\hline IM & & 19,88 & 19,07 & 20,24 & 23,57 & 24,06 & 23,84 & 28,13 & 28,21 & 30,54 \\
\hline
\end{tabular}

Valores medios seguidos de letras diferentes en la misma fila indican diferencias significativas entre tratamientos para ese parámetro de acuerdo con el test de Duncan $(P>0,05)$. La ausencia de letras indica que no es significativo.

Crisosto et al. (2003) indicaron que las tres características principales de aceptación de la cereza eran el color, los sólidos solubles totales y la acidez titulable. El poder contar con una relación que englobe a los tres factores puede ser interesante de cara a estimar su comportamiento de mercado. En "Prime Giant" se encontró una relación polinómica entre el matiz, color de la piel de las cerezas, y la concentración de sólidos solubles totales y a su vez con la acidez titulable, siendo éstas de sentido opuesto (Figura 3). Estas relaciones, muy similares a las descritas por Yommi et al. (2012) para las variedades Lapins y Sweetheart, pueden resultar de interés para determinar el momento adecuado de la cosecha, así como la evolución de SST y AT en refrigeración, en función del color de las cerezas.

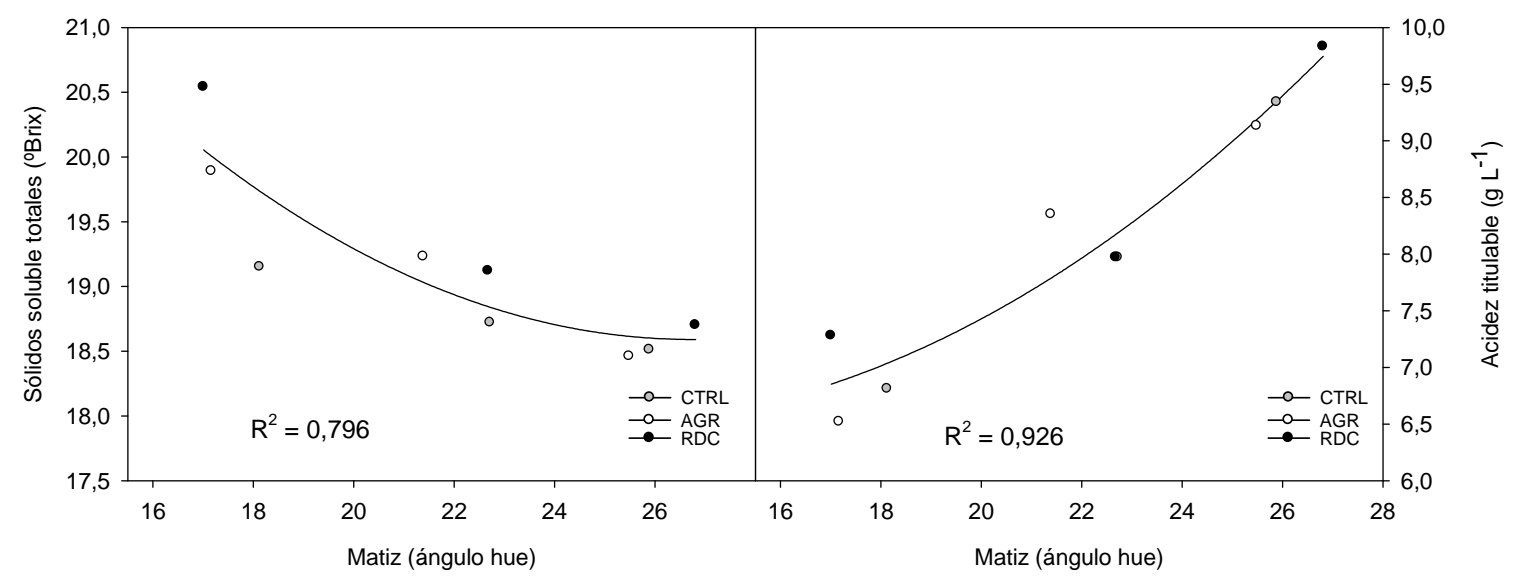

Figura 3. Relación entre el matiz (ángulo hue) y los sólidos soluble totales ( ${ }^{\circ} \mathrm{Brix}$ ) y la acidez titulable $\left(\mathrm{g} \mathrm{L}^{-1}\right)$.

\section{4- Conclusiones y recomendaciones}

El déficit hídrico ligero aplicado en precosecha no afectó negativamente a la calidad en ninguno de los tres momentos de evaluación: cosecha, frigo-conservación y comercialización. Incluso, en el momento de cosecha se dieron valores más altos de acidez 
titulable y matiz en RDC que en AGR. Independientemente de la inexistencia de diferencias significativas en volumen de fruto y firmeza, se observó una alta correlación entre ellas y el agua aplicada. Tanto los sólidos solubles totales como la acidez titulable presentaron buenas correlaciones con el matiz, las que pueden ser de interés de cara a determinar el momento de cosecha. Las pérdidas de peso durante la conservación no dieron lugar a diferencias entre tratamientos. Sin embargo, RDC presentó una tendencia a menores pérdidas de peso, característica que por su interés deberá ser comprobada en sucesivas cosechas. Ahorros de agua precosecha en RDC del $17 \%$ y $35 \%$ respecto a CTRL y AGR, respectivamente no afectaron a la producción de ninguna de las dos recolecciones ni a la total $\left(15,5 \mathrm{t} \mathrm{ha}^{-1}\right)$.

Teniendo en cuenta que son los primeros resultados obtenidos tras un único año de aplicación del déficit hídrico ligero en precosecha de "Prime Giant", es pronto para recomendar su aplicación a nivel comercial, de ahí el interés de poder validarlos en las siguientes campañas.

\section{5.- Agradecimientos}

Este trabajo fue financiado por el Ministerio de Economía y Competitividad (MINECO) y está encuadrado en el proyecto: 'Evaluación de la automatización del riego deficitario en frutales. Mejora de la gestión del agua para asegurar la productividad y sostenibilidad'. Referencia: AGL2013-49047-C2-1R.

\section{6- Bibliografía}

Conesa, M.R., De la Rosa, J.M., Artés-Hernández, F., Dodd, I.C., Domingo, R., Pérez-Pastor A. 2014. Long-term impact of deficit irrigation on the physical quality of berries in 'Crimson Seedless' table grapes. J. Sci. Food Agric. 95 (12), 2510-2520.

Crisosto, C.H., Crisosto, G.M., Ritenour M.A. (2002) Testing the reliability of skin colour as an indicator of quality for early season Brooks (Prunus avium L.) cherry. Postharvest Biology \& Technology, 24, 147-154.

Crisosto, C. H., Crisosto, G. M., Metheney, P. (2003) Consumer acceptance of "Brooks" and "Bing" cherries is mainly dependent on fruit SSC and visual skin color. Postharvest Biology \& Technology, 28, 159-167.

De la Rosa, J.M., Domingo, R., Gómez-Montiel, J., Pérez-Pastor, A. (2015). Implementing deficit irrigation scheduling through plant water stress indicators in early nectarine trees. Agricultural Water Management. 152, 207-216.

Díaz-Mula, H.M., Castillo, S., Martínez-Romero, D., Valero, D., Zapata, P.J., Guillén, F., Serrano, M. (2009) Organoleptic, nutritive and functional properties of sweet cherry as affected by cultivar and ripening stage. Food. Sci. Technol. Int, 15, 535-544.

Domingo, R., Ruiz-Sánchez, M.C., Sánchez-Blanco, M.J., Torrecillas, A. (1996). Water relations, growth and yield of Fino lemon trees under regulated deficit irrigation. Irrigation Sci. 16, 115-123.

Drake S.R. and Elfving D.C. (2002). Indicators of maturity and storage quality of 'Lapins' sweet cherry. Hort Technology. 12, 687-690.

Egea, G., Nortes, P.A., Domingo, R., Baille, A., Pérez-Pastor, A., González-Real, M.M. (2013). Almond agronomic response to long-term deficit irrigation applied since orchard establishedment. Irrigation Sci. 31, 445-454.

Falagán, N., Artés, F., Gómez, P.A., Artés-Hernandez, F., Conejero, W., Aguayo E. (2015) Deficit irrigation strategies enhance health-promoting compounds through the intensification of specific enzymes in early peaches. Journal of the Science of Food and agriculture, 96 (5), 1803-1813.

Fereres, E y Castel, J.R. (1981). Drip irrigation management. Division of Agricultural Sciences, University of California. Publicación Leaflet 21259. 
Gonçalves, B., Silva, A. P., Moutinho-Pereia, J., Bacelar, E., Rosa, E., Meyer, A. S. (2007) Effect of ripeness and postharvest storage on the evolution of color and anthocyanins in cherries (Prunus avium L.). Food Chem., 103, 976-984.

Marsal, J., López, G., del Campo, J., Mata, M., Arbones, A., Girona J. (2010). Postharvest regulated deficit irrigation in 'Summit' sweet cherry: fruit yield and quality in the following season. Irrigation Sci. 28, 181-189.

Pérez-Pastor, A., Ruiz-Sánchez, M.C., Martínez, J.A., Nortes, P.A., Artés, F., Domingo, R. (2007) Effect of deficit irrigation on apricot fruit quality at harvest and during storage. J Sci Food Agric., 87, 2409-2415.

Ruiz-Sánchez, M.C., Domingo, R., Castel J.R. (2010) Deficit irrigation in fruit trees and vines in Spain: a review. Span. J. Agric. Res., 8 (S2), S5-S20.

Serrano, M., Diaz-Mula, H.M., Zapata, P.J., Castillo, S., Guillén, F., Martinez-Romero, D., Valverde Juan, M., Valero, D. (2009) Maturity stage at harvest determines the fruit quality and antioxidant potential after storage of sweet cherry cultivars. J. Agric. Food Chem., 57, 3240-3246.

Shackel K.A., Ahmadi H., Biasi W., Buchner R., Goldhamer D.A. et al. (1997) Plant water status as an index of irrigation need in deciduous fruit trees. Hort. Technol. 7, 23-29.

Yommi, A., Fernandez, V., Guerra, N., Cendoya, G., Quillehauquy, V. (2012) Skin colour changes in fruits of two sweet cherry cultivars, its heterogeneity and relationship with quality indices. Acta horticulturae, 934, 1055-1061. 